\title{
OBTENCIÓN DE UN FILTRANTE DE MAIZ MORADO (Zea mays L.), EVALUACIÓN DE PÉRDIDA DE COLOR Y DEGRADACIÓN DE ANTOCIANINAS EN EL ALMACENAJE
}

\author{
OBTAINING A FILTER PURPLE CORN (Zea mays L.), EVALUATION OF FADING AND \\ DEGRADATION IN STORAGE ANTHOCYANINS
}

\author{
${ }^{1}$ Diana Nolazco C. y ${ }^{2}$ Miguel Araujo V.
}

\begin{abstract}
Resumen
El trabajo de investigación permitió obtener un filtrante de maíz morado, evaluar la pérdida del color y degradación de las antocianinas durante el almacenaje a 70, 80, 90 y $100 \%$ de humedad relativa (H.R.). Se obtuvo el filtrante de maíz morado mediante una mezcla de canela $1 \%$, clavo de olor $1 \%$, grano $10 \%$ y coronta de maíz morado $88 \%$, con una tamaño de partícula entre 2 y $4 \mathrm{~mm}$. La caracterización del filtrante reportó: humedad 7.9\%, carbohidratos 74.4\%, cenizas $2.4 \%$, grasa $0.2 \%$, proteína $5.1 \%$, fibra $10.0 \%$, actividad de agua 0.48 ; y coordenadas de color $\mathrm{L}^{*}=40.26$, $\mathrm{a}^{*}=7.96 \mathrm{y} \mathrm{b}=0.82$. Las pruebas estadísticas reportaron mayor estabilidad en: color y antocianinas monoméricas en los filtrantes almacenados en ambientes a 70 y $80 \%$ de humedad relativa (H.R.). La cinética de degradación fue de primer orden con velocidades de reacción de $0.0126,0.0149,0.0283$ y 0.0291 días $^{-1}$, durante el almacenaje a 70, 80, 90 y $100 \%$ de humedad relativa (H.R.), respectivamente. El tiempo de vida media fue de 55, 46.5, 24.5 y 23.8 días a $70,80,90$ y $100 \%$ de humedad relativa (H.R.), respectivamente en empaques de polipropileno con permeabilidad de 0.705 g-milipulgadas/ $\mathrm{m}^{2}$-día-mmHg.
\end{abstract}

Palabras Claves: antocianinas, color, degradación, maíz morado.

\section{Summary}

This research allowed the production of a filtering purple corn infusion bags, data of the loss of color and degradation of anthocyanins during their storage at 70, 80, 90 and 100\% relative humidity (RH). It was obtained a purple corn infusion bags by a mixture of $1 \%$ cinnamon, $1 \%$ clove, $10 \%$ corn grains and $88 \%$ purple maize cobs; all of them with a particle size between 2 and $4 \mathrm{~mm}$. The characterization of infusion bags reported: moisture 7.9\%, carbohydrate $74.4 \%$, ash $2.4 \%$, fat $0.2 \%$, protein $5.1 \%$, fiber $10.0 \%$, water activity 0.48 ; and color coordinates $\mathrm{L} *=40.26, *=7.96 \mathrm{~b} *=0.82$. The results reported a statistical higher stability in: color and monomerics anthocyanins in the infusion bags stored in environments at 70 and $80 \%$ relative humidity (RH). The degradation kinetics was first order during storage at 70,80 , 90 and $100 \%$ relative humidity $(\mathrm{RH})$, with speeds of reaction of $0.0126,0.0149,0.0283$ and 0.0291 day $^{-1}$, respectively. The half-life bags was 55, 46.5, 24.5 and 23.8 days stored at 70, 80, 90 and 100\% relative humidity (RH), respectively in polypropylene bags with permeability 0,705 g-milipulgadas/m2-day- $\mathrm{mmHg}$.

Key words: anthocyanins, color, degradation, corn purple

\section{Introducción}

En los últimos años el maíz morado ha despertado gran interés para la industria alimentaria, cosmetológica y farmacéutica debido a su alto contenido en antocianinas. Estos colorantes naturales imparten color y alta capacidad antioxidante al ser consumidos, encontrándose en mayor proporción en la coronta del maíz morado y en menor proporción en el pericarpio del grano (Araujo, 1995).

La industria de alimentos busca desarrollar hoy en día productos variados que no sólo busquen satisfacer a los consumidores sino también que sean de fácil procesamiento y con una mínima maquinaria a nivel industrial, que podría incrementar las exportaciones y las áreas de producción. Por lo expuesto se decidió llevar a cabo la presente investigación planteando los siguientes objetivos:

- Obtención de un filtrante de maíz morado

- Evaluación de la pérdida de color y degradación de antocianinas en el filtrante de maíz morado durante el almacenaje a 70, 80, 90 y $100 \%$ de humedad relativa (H.R.).

\section{Materiales y métodos}

\subsection{Lugar de ejecución}

El presente trabajo de investigación se llevó acabo en las instalaciones de la Universidad Nacional Agraria La Molina. Las pruebas experimentales se realizaron en los 
Laboratorios de Análisis de Alimentos, Microbiología y Planta Piloto de Tecnología de Alimentos y Productos Agropecuarios (TAPA).

\subsection{Materia prima e insumos}

Maíz morado proveniente de las localidades de Canta y Arequipa adquiridas en el mercado mayorista de Lima; canela y clavo de olor. Amonio Cuaternario e hipoclorito de sodio como desinfectante.

\subsection{Materiales, Reactivos y Equipos}

Mesas de acero inoxidable, cuchillos, botellas de color ámbar y tapas, campanas desecadoras, agitador magnético, potenciómetro digital, balanza analítica, balanza digital, colorímetro, espectrofotómetro, selladora de bolsas manual, secador de túnel por aire caliente, molino de martillos, molino de mortero, estufa, mufla, medidor de actividad de agua. Bolsas de polipropileno, papel filtro termosellable, tamices, micrómetro, ácido sulfúrico, cloruro de litio, cloruro de magnesio, dicromato de sodio, nitrito de sodio, cloruro de sodio, cromato de potasio, silica gel.

\subsection{Método de Análisis}

\subsubsection{Análisis Fisicoquímicos}

-Humedad, grasa, proteína, fibra, cenizas. Por el método de la A.O.A.C (1997).

Carbohidratos. Por diferencia, esto es el 100\% menos el resultado de análisis anteriores (A.O.A.C, 1990).

Sólidos Totales. Método recomendado por Lees (1969). pH. Por el método potenciómetrico (A.O.A.C, 1990).

Color. Mediante el colorímetro marca Minolta CR400 (A.O.A.C, 1995).

Curvas de secado. Según las recomendaciones de Casp y Abril (1999); citados por Cancino (2003).

Isoterma de Sorción. Método recomendado por Mattos (1999); mencionado por Cancino (2003).

Antocianinas monoméricas. Por el método espectrofotométrico del $\mathrm{pH}$ diferencial recomendado por Fuleki y Francis (1968) modificado por Giusti y Wrolstad (2001).

Determinación de la cinética de degradación. Se determinó orden de la reacción, constante de velocidad $(\mathrm{k})$ y tiempo de vida media $\left(\mathrm{t}^{1 / 2}\right)$, de acuerdo a lo recomendado por Labuza (1979).

\subsubsection{Análisis Físicos}

-Rendimientos promedios. Permeabilidad del Empaque. Por el método del desecante, descrito por la ASTM (1984); recomendado por Espinoza (1995). Espesor de la Película Flexible, por el método del micrómetro descrito por la ASTM Standard (1984); recomendado por Espinoza (1995).

\subsubsection{Análisis Sensorial y Estadístico}

Se realizó en las siguientes etapas de la investigación:

Para determinar porcentaje de grano y de coronta en el filtrante de maíz morado

Se realizó una evaluación sensorial mediante el uso de una prueba de Preferencia Ampliada evaluándose la característica de sabor en muestras de chicha morada a un panel de 30 jueces no entrenados. El objetivo fue determinar el porcentaje de grano y coronta de maíz morado de mayor preferencia en las muestras de filtrante. Los resultados fueron evaluados estadísticamente mediante la prueba no paramétrica de Friedman.

Para determinar el tamaño de partícula de las materias primas antes del envasado

Se realizó una evaluación sensorial mediante la prueba de preferencia ampliada evaluando la característica de aspecto general en las tres muestras de chicha morada, el panel fue de 30 jueces. El objetivo fue determinar el tamaño de partícula adecuado de las materias primas del filtrante evitando precipitados en la bebida final. Los resultados fueron evaluados estadísticamente mediante la prueba no paramétrica de Friedman.

\section{Almacenamiento del filtrante de maíz morado}

Los resultados de los análisis de variación de: antocianinas monoméricas, humedad y actividad de agua de los filtrantes almacenados a $70,80,90$ y $100 \%$ de humedad relativa (H.R.), respectivamente; fueron evaluados estadísticamente mediante un Experimento Factorial conducido por un DBCA a un nivel de significancia del $5 \%$, los resultados en donde se encontró diferencias significativas se les comparó entre sí mediante la Prueba de Comparación de Tukey, descrito por Calzada (1982).

Para evaluar el \% de humedad relativa (H.R.) de almacenaje más adecuado para mantener lo más estable posible el contenido de antocianinas se realizó la Prueba de Comparación de Tukey comparando el contenido promedio de antocianinas monoméricas a los 12 y 24 días de almacenaje a las 4 diferentes humedades relativas $(70$, 80,90 y $100 \%$ de H.R.).

Determinación de parámetros cinéticos de la degradación de antocianinas monoméricas en el filtrante de maíz morado

Para ajustar los datos experimentales, determinar el orden y velocidad de la reacción se utilizó la regresión lineal simple.

\subsection{Metodología Experimental}

2.5.1 Descripción de las operaciones para la obtención de un filtrante de Maíz Morado

En la Fig. 1 se presenta el Flujo Cualitativo de Operaciones para la obtención de un filtrante de Maíz Morado. 


\section{Selección}

Esta operación se realizó manualmente, separando así las corontas de maíz morado, canela y clavo de olor que presentaron signos de deterioro o algún daño.

\section{Desgranado}

El desgranado del maíz morado se realizó en forma manual.

\section{Lavado y desinfectado}

La coronta, los granos, la canela y el clavo de olor fueron lavados con agua a presión facilitando la eliminación de la suciedad y de las impurezas presentes. Para la desinfección las materias primas fueron sumergidas en una solución de hipoclorito de sodio a 100 ppm de C.L.R por 5 minutos y posteriormente en amonio cuaternario al $0.2 \%$ por 10 minutos

\section{Trozado o cortado}

Se realizó un trozado a la coronta y canela con la finalidad de aumentar la superficie de contacto entre el aire caliente y la materia prima. El tamaño de partícula fue aproximadamente de 3 a $5 \mathrm{~cm}$.

\section{Secado}

Se realizó por separado a cada materia prima en un secador de túnel por aire caliente a una temperatura de 60 a $65^{\circ} \mathrm{C}$ y una velocidad de aire promedio de $2.5 \mathrm{~m} / \mathrm{s}$. La finalidad fue eliminar el hasta una humedad final entre $5-8 \%$.

\section{Mezclado}

Se realizó la mezcla de canela, clavo de olor, coronta y grano de maíz en proporciones adecuadas, para luego proceder a la molienda.

\section{Molienda}

La molienda se realizó con ayuda de un molino de martillos con cuchillas de donde se obtuvo el tamaño de partícula adecuado.

\section{Tamizado}

Se procedió a utilizar tamices para obtener un tamaño de partícula uniforme.

\section{Envasado}

El envasado se realizó en bolsitas filtrantes de $15 \mathrm{~g}$ cada uno, usando papel termosellable de $7 \mathrm{~cm}$. de ancho y $10 \mathrm{~cm}$. de largo, posteriormente se envasó en bolsas de polipropileno. El rendimiento de cada bolsita es de 1 litro de chicha morada.

\section{Almacenaje}

Se realizó el almacenaje de los filtrantes a 70, 80, 90 y $100 \%$ de humedad relativa (H.R.) de donde fue extraído de acuerdo al avance de la investigación.

\subsubsection{Esquema Experimental}

En la Fig. 2 se presenta el esquema experimental que se llevó a cabo en la presente investigación.

\section{Caracterización de las materias primas}

- Composición fisicoquímica de canela, clavo de olor, coronta y grano de maíz morado

La canela, clavo de olor, coronta y grano de maíz morado fueron caracterizados en: humedad, grasa, proteínas, cenizas, fibra y carbohidratos.

\section{- Rendimientos promedios}

Se determinó los rendimientos promedios de coronta y grano de maíz morado con respecto a la materia prima entera (expresados en \%).

\section{- Curvas de Secado}

Las materias primas fueron secadas por medio de un túnel de aire caliente a una temperatura entre 60 y $65^{\circ} \mathrm{C}$, a una velocidad de aire promedio de $2.5 \mathrm{~m} / \mathrm{s}$. Luego se graficó la variación de humedad vs. tiempo de secado para cada materia prima.

\section{Porcentajes de Grano y Coronta en el filtrante de maíz morado}

Se procedió a mezclar la canela, el clavo de olor, los granos y la coronta en las siguientes proporciones: 1:1:10:88, 1:1:15:83 y $1: 1: 20: 78$, respectivamente.

Tamaño de partícula de canela, clavo, grano y coronta de maíz morado

Se procedió a realizar la molienda de canela, clavo, grano y coronta de maíz morado en tamaños de partículas de: 1,2 y $4 \mathrm{~mm}$.

\section{Características del empaque}

Se procedió a determinar: permeabilidad al vapor de agua y espesor del material.

\section{Caracterización Fisicoquímica del filtrante de maíz morado}

Se caracterizó fisicoquímicamente el filtrante de maíz morado en: carbohidratos, proteína, grasa, fibra, cenizas y humedad. Se realizó en el producto la determinación de: color, actividad de agua e isoterma de absorción. 


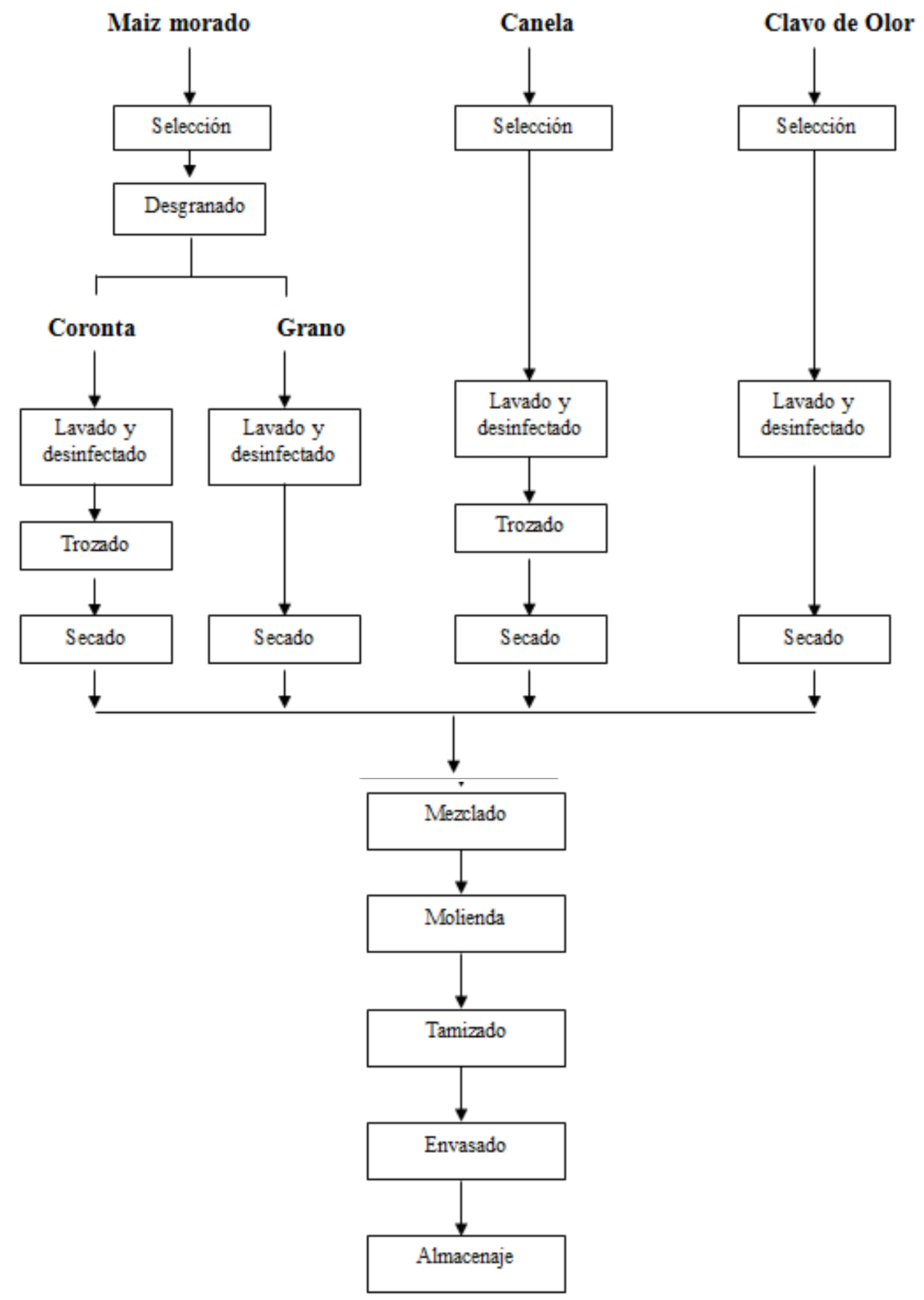

Figura 1. Flujo cualitativo de operaciones para la obtención de un filtrante de maíz morado. 


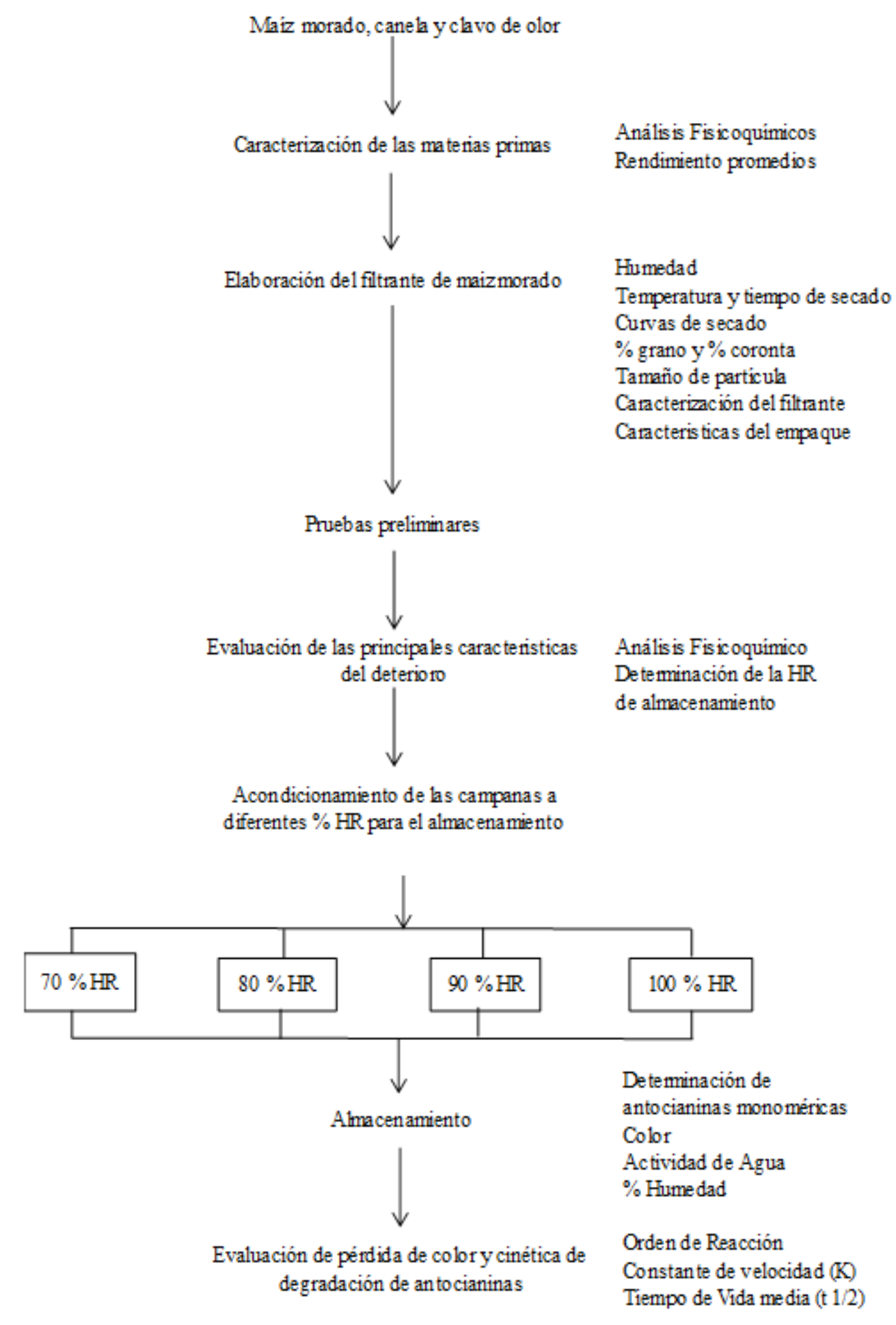

Figura 2. Esquema experimental para obtención de un filtrante de maíz morado, evaluación del color y degradación de antocianinas en el almacenaje. 


\section{Almacenamiento del filtrante de maíz morado}

-Los filtrantes de maíz morado se sometieron a almacenamiento a temperatura ambiente $\left(20{ }^{\circ} \mathrm{C}\right)$ y a diferentes condiciones de humedad relativa (H.R.): $70 \%, 80 \%, 90 \%$ y $100 \%$; manteniendo constante las condiciones del empaque.

-Para las evaluaciones del color se registraron las medidas de color en las coordenadas $L^{*}, a^{*}$ y b* durante el almacenaje.

-Para determinar el comportamiento de degradación de antocianinas monoméricas en los filtrantes se determinó la concentración del colorante cada 6 días a 90 y $100 \%$ de humedad relativa (H.R.) y cada 12 días en los filtrantes almacenados a 80 y $70 \%$ de humedad relativa (H.R.). Se registraron las variaciones del \% de humedad y de actividad de agua durante el almacenaje como factores importantes de la degradación de color y de antocianinas en el producto.

\section{Determinación de parámetros cinéticos de degradación de antocianinas en el filtrante de maíz morado}

El orden de la reacción de degradación de las antocianinas en el filtrante de maíz morado se determinó graficando la variación de la concentración de antocianinas vs. el tiempo de almacenaje, la representación en coordenadas lineales, semilogaritmicas y de 1/A, que presentó un coeficiente de correlación superior a 0.85 correspondería a una reacción de orden 0 , primer orden y segundo orden, respectivamente. Posteriormente se determinó la velocidad de la degradación $(\mathrm{k})$, correspondiente a la pendiente de la recta obtenida entre la concentración de antocianinas y el tiempo de almacenaje; y el tiempo de vida media se determinó según el orden de la reacción obtenida, siendo: (Ln 2)/k y 1/([A] $]_{0}$ ) para reacciones de primer y segundo orden, respectivamente.

\section{Resultados y discusión}

\subsection{Caracterización de las materias primas}

3.1.1 Composición fisicoquímica de la canela, el clavo de olor, la coronta y el grano de maíz morado

El contenido de humedad, proteínas, fibra, grasa, carbohidratos y cenizas se muestran en el Tabla 1 .

\subsubsection{Rendimiento promedio de Coronta y Grano de Maíz Morado}

El rendimiento promedio de la coronta fue de $20.55 \%$ y grano de maíz morado 79.44\%; similares a los resultados obtenidos por Fernández (1995), quien encontró 20.79\% y $79.21 \%$ para la coronta y grano, respectivamente.

Tabla 1. Composición fisicoquímica de la canela, clavo de olor, coronta y grano de maíz morado.

\begin{tabular}{|c|c|c|c|c|c|c|c|c|}
\hline \multirow{3}{*}{ Componentes } & \multicolumn{8}{|c|}{ Valores reportados $(\%)$} \\
\hline & \multicolumn{2}{|c|}{ Canela } & \multicolumn{2}{|c|}{ Clavo de olor } & \multicolumn{2}{|c|}{ Coronta } & \multicolumn{2}{|c|}{ Grano } \\
\hline & b.h & b.s & b.h & b.s & b.h & b.s & b.h & b.s \\
\hline Humedad & 10.8 & 12.1 & 25.3 & 33.9 & 30.4 & 43.7 & 28.4 & 39.7 \\
\hline Proteínas (Fc 6.25) & 3.3 & 3.7 & 6.3 & 8.4 & 2 & 2.9 & 6.3 & 8.8 \\
\hline Fibra & 36.5 & 0.4 & 7.4 & 9.9 & 16.5 & 23.7 & 0.7 & 1.0 \\
\hline Grasa & 0.3 & 0.3 & 3.2 & 4.3 & 0.0 & 0.0 & 2.2 & 3.1 \\
\hline Carbohidratos & 49.1 & 55 & 57.8 & 0.8 & 49.5 & 71.1 & 61.2 & 85.5 \\
\hline Cenizas & 3.4 & 3.8 & 4.4 & 5.9 & 1.6 & 2.3 & 1.2 & 1.7 \\
\hline
\end{tabular}

\subsection{Curvas de Secado}

En las curvas de secado obtenidas, se logró observar que el grano de maíz morado no mostró una pérdida de humedad rápida en comparación a la coronta, clavo de olor y canela. Las humedades finales expresadas en \% b.h fueron: canela $5.6 \%$, clavo de olor $5.2 \%$, coronta $5 \%$ y grano $5.5 \%$. Cheftel y Cheftel (1976) indica que una concentración elevada de constituyentes polímeros como proteínas, almidones y azúcares amorfos aumenta la proporción de agua ligada y prolonga la segunda fase de secado.

\subsection{Porcentaje de grano y coronta en el filtrante de maíz morado}

Se encontró diferencias significativas para todas las muestras de filtrantes en cuanto al sabor, encontrando que la muestra de mayor preferencia era la proporción: canela $1 \%$, clavo $1 \%$, grano $10 \%$ y coronta $88 \%$.

3.4Tamaño de partícula de canela, clavo, grano y coronta de maíz morado

A un nivel de significancia de $95 \%$ se encontraron diferencias significativas entre las tres muestras de filtrante con tamaños de 1, 2 y $4 \mathrm{~mm}$. Los jueces determinaron el primer lugar en preferencia a la muestra con tamaño de partícula de $4 \mathrm{~mm}$, y en segundo lugar a la muestra con $2 \mathrm{~mm}$; teniendo en cuenta los resultados se decidió optar por una molienda con tamaños de partículas de $2 \mathrm{~mm}$ como mínimo y $4 \mathrm{~mm}$ como máximo. 


\subsection{Características del empaque}

La permeabilidad al vapor de agua fue de 0.705 g-milipulgada/ $\mathrm{m}^{2}$-día-mmHg con un espesor del material de polipropileno de 1.3 milipulgadas. El valor se encuentra dentro del rango 0.703 - 0725 g-milipulgada/ $\mathrm{m}^{2}$-día-mmHg para películas de polipropileno, reportado por Espinoza (1995).

\subsection{Caracterización del filtrante de maíz morado}

\subsubsection{Composición Fisicoquímica}

La composición fisicoquímica del filtrante de maíz morado fue: humedad $7.9 \%$, carbohidratos $74.4 \%$, cenizas $2.4 \%$, grasa $0.2 \%$, proteína $5.1 \%$ y fibra $10.0 \%$.

Tabla 2. Composición fisicoquímica del filtrante de maíz morado.

\begin{tabular}{lcc}
\hline \multicolumn{1}{c}{ Componentes } & \multicolumn{2}{c}{ Valores reportados (\%) } \\
& b.h & b.s \\
\hline Humedad & 7.90 & 8.60 \\
Carbohidratos & 74.40 & 91.60 \\
Cenizas & 2.40 & 2.60 \\
Grasa & 0.20 & 0.22 \\
Proteína (factor: 6.25$)$ & 5.10 & 5.50 \\
Fibra & 0.00 & 10.90 \\
\hline
\end{tabular}

\subsubsection{Determinación de Actividad de Agua y} Coordenadas de Color

La actividad de agua del filtrante fue de 0.48 . El valor de la coordenada $L^{*}=40.26$ expresa la tendencia a un color oscuro, el valor $\mathrm{a}^{*}=7.96 \mathrm{y} \mathrm{b}^{*}=0.82$ representan la tendencia al rojo y morado, respectivamente.

\subsubsection{Isoterma de Adsorción}

La isoterma ajustada por GAB se muestra en la Fig. 3. Como se puede observar se obtuvo una isoterma del tipo II, de forma sigmoidea o tipo S, de las cinco establecidas por Van der Waals, las cuales son las mas frecuentes en alimentos (Martínez et al., 1998; mencionado por Vega et al., 2006).

Mediante la ecuación de GAB se obtuvo la siguiente expresión matemática:

$\mathrm{Aw} / \mathrm{m}=0.191+16.203 \mathrm{Aw}-14.565 \mathrm{Aw} 2$

La expresión relaciona la actividad de agua y el valor monocapa en el filtrante de maíz morado. Se obtuvo un valor monocapa de $0.0528 \mathrm{~g}$ agua/g m.s correspondiente a una Aw de 0.10 .

\subsection{Almacenamiento del Filtrante de Maíz morado}

\subsubsection{Evaluación del color (coordenadas a*, b* y L*)}

En el Tabla 3 se muestra los resultados obtenidos de las coordenadas $a^{*}, b^{*}$ y L* durante el almacenaje 70,80 , 90 y $100 \%$ de humedad relativa (H.R.), respectivamente.

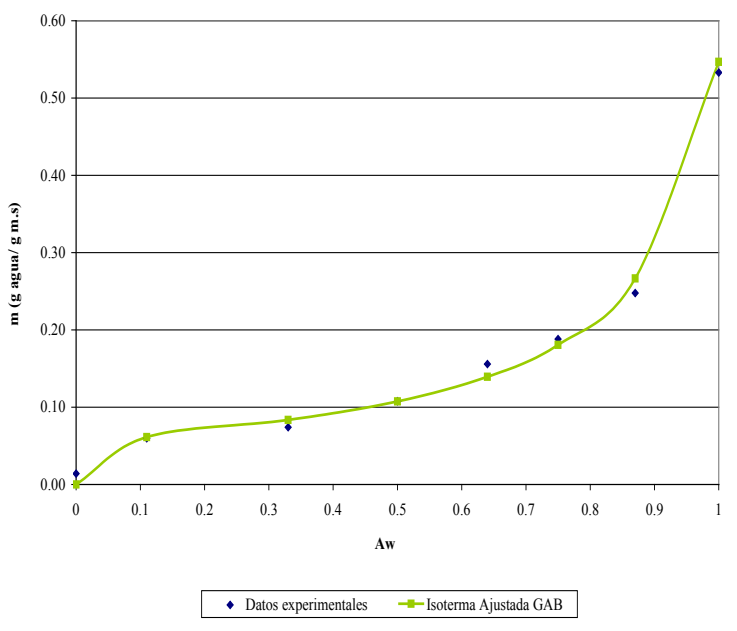

Figura 3. Isoterma de sorción del filtrante de maíz morado a $20^{\circ} \mathrm{c}$ según gab.

Tabla 3. Variación de las coordenadas $a^{*}, b^{*}$ y $1^{*}$ promedio de los filtrantes almacenados bajo diferentes humedades relativas $(\mathrm{hr})$.

\begin{tabular}{cccc}
\hline $\begin{array}{c}\text { Humedad Relativa } \\
\text { (H.R) }\end{array}$ & $\mathrm{a}^{*}$ & $\mathrm{~b}^{*}$ & $\mathrm{~L}^{*}$ \\
\hline Inicial & 7.96 & 0.82 & 40.26 \\
$70 \%$ & 8.06 & 0.77 & 42.06 \\
$80 \%$ & 8.80 & 0.89 & 42.06 \\
$90 \%$ & 8.39 & 1.10 & 46.50 \\
$100 \%$ & 8.17 & 1.12 & 42.17 \\
\hline
\end{tabular}

En la coordenada $\mathrm{a}^{*}$ mostró tendencia del color morado al rojizo, en $b^{*}$ la tendencia fue al amarillo, y en $L^{*}$ la tendencia fue a un color claro.

\subsubsection{Degradación de antocianinas monoméricas}

En la Fig. 4 se presenta la variación del contenido de antocianinas monoméricas $(\mathrm{mg} / \mathrm{L})$ con respecto al tiempo de almacenaje a 70, 80, 90 y $100 \%$ de humedad relativa (H.R.), con una tendencia a un decrecimiento exponencial, donde se presenta una fuerte caída al inicio del almacenaje pero se hace mas lento con el tiempo y teóricamente nunca se llega hasta un valor de cero (Núñez y Chumbiray, 1991; mencionados por Martínez, 2004). Por otro lado, la evaluación estadística no encontró diferencias significativas en el cambio de la concentración de antocianinas entre las muestras almacenadas a 70 y $80 \%$ de humedad relativa (H.R) a los 12 días y entre 90 y $100 \%$ de humedad relativa (H.R) a los 24 días de almacenaje; es decir la mayor estabilidad en la concentración del colorante en los filtrantes de maíz morado se obtiene a 70 o $80 \%$ de humedad relativa (H.R). La disminución a la mitad de la concentración de antocianinas monoméricas en las bolsas filtrantes de maíz morado se dio entre los 24 y 30 días a 90 y $100 \%$ 
de humedad relativa (H.R), y entre los 48 y 60 días en almacenamientos a 70 y $80 \%$ de humedad relativa (H.R), respectivamente; es decir el tiempo de reducción del contenido de antocianinas monoméricas a la mitad se incrementa el doble a medida que se disminuye en un $20 \%$ la humedad relativa (H.R.) del ambiente de almacenaje.

La variación del \% de humedad promedio de los filtrantes almacenados se pueden observar en la Fig. 5.

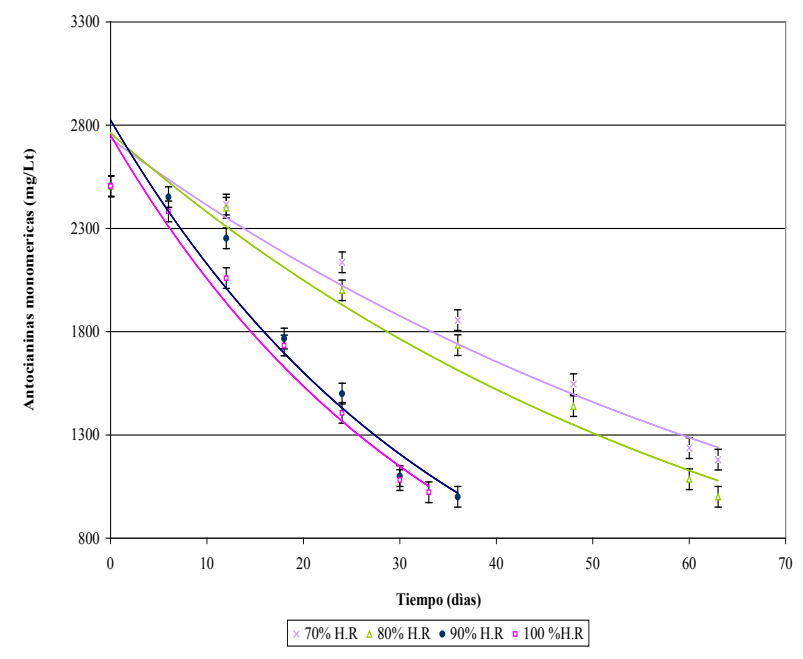

Figura 4. Variación del contenido de antocianinas monoméricas con respecto al tiempo de almacenaje bajo diferentes humedades relativas (h.r.).

Las humedades finales en el producto en $\%$ b.h. fueron: $16.35,16.48,16.64$ y $16.97 \%$ para almacenamientos a 70, 80, 90 y $100 \%$ de humedad relativa (H.R.). Los resultados de la evaluación estadística al $5 \%$, encontraron mayores diferencias en cuanto a los cambios de humedad en los filtrantes de maíz morado almacenados a $100 \%$ de humedad relativa (H.R.). Robertson (1993) refiere que en alimentos semisecos la ganancia de humedad durante el almacenamiento es el resultado final de dos mecanismos acoplados: el pasaje de humedad a través del envase y la absorción de la misma por parte del alimento. En la Fig. 6 se presentan las variaciones en los valores de actividad de agua registrados en el almacenaje. Se observó que los valores de actividad de agua se incrementaron desde 0.48 hasta $0.699,0.800,0.816,0.824$ para las muestras almacenadas a 70, 80, 90 y 100\% humedad relativa (H.R), respectivamente.

Los resultados estadísticos $(\alpha=0.05)$ encontraron mayores diferencias significativas durante el tiempo de almacenaje entre los filtrantes a 90 y $100 \%$ de humedad relativa (H.R) en comparación con las almacenadas a 70 y $80 \%$ de humedad relativa (H.R), presentando los últimos mayor estabilidad.

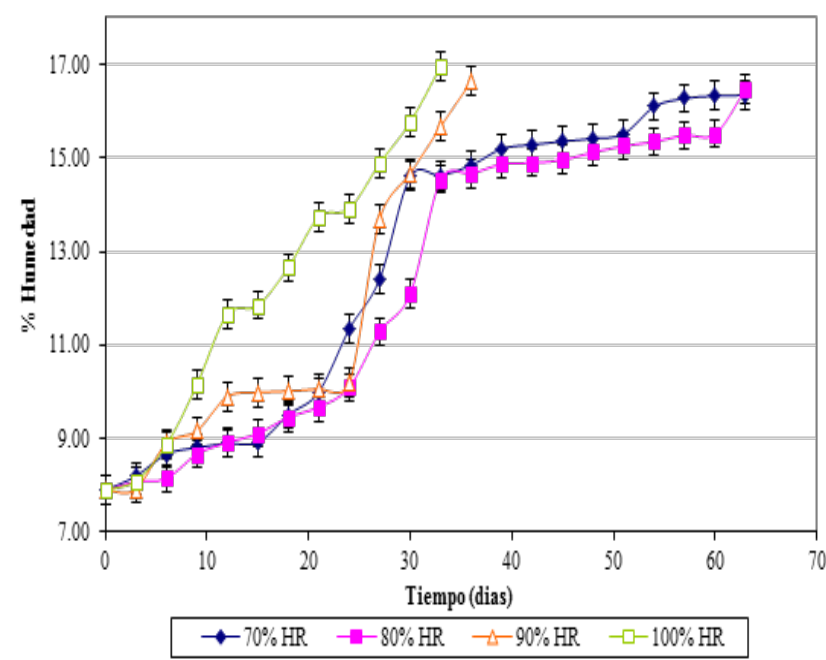

Figura 5. Variación del \%humedad promedio de los filtrantes almacenados bajo diferentes humedades relativas (h.r.).

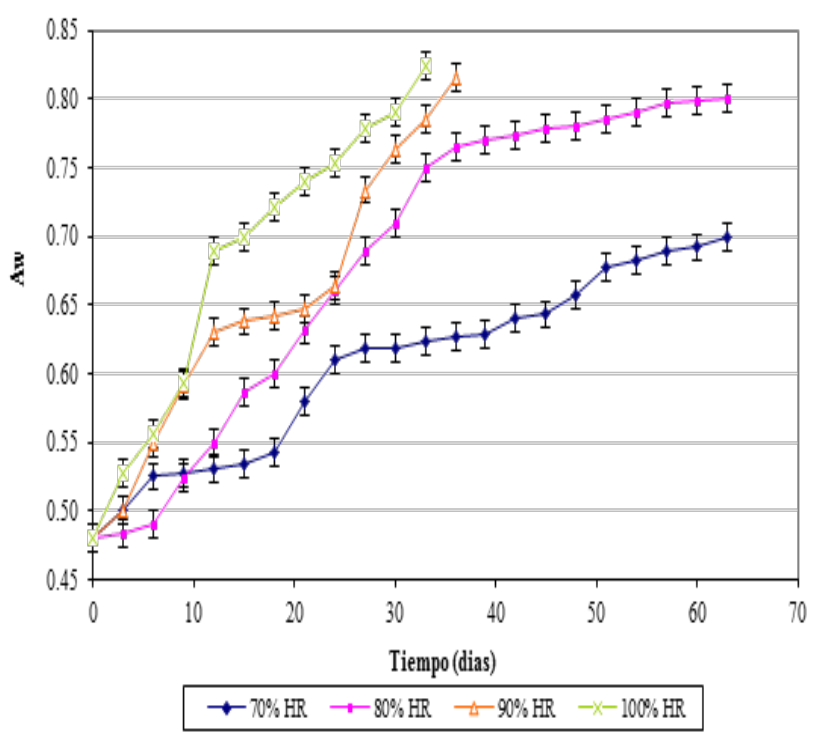

Figura 6. Variación de la actividad de agua promedio de los filtrantes de maíz morado almacenados bajo diferentes humedades relativas (h.r.).

\section{A. Determinación del orden de reacción}

En la Fig. 7 se presenta la relación entre el Ln de la concentración de antocianinas monoméricas vs. el tiempo (días). Según los resultados existe una relación lineal entre el Ln de la concentración de antocianinas con respecto al tiempo, esta relación establece un modelo de primer orden (Fennema, 2000). 


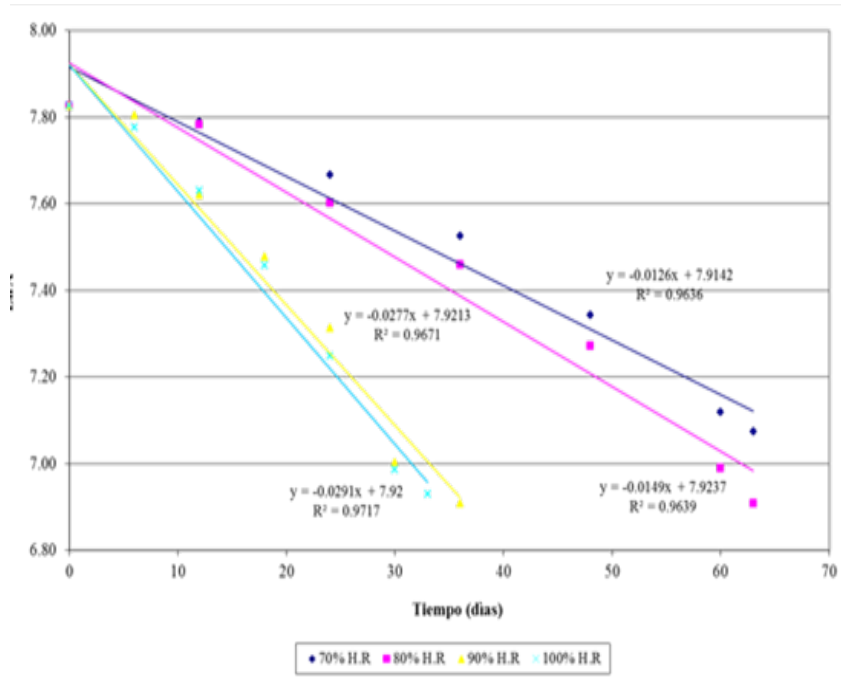

Figura 7. Relación lineal entre ln de antocianinas y el tiempo de almacenaje de filtrantes bajo diferentes humedades relativas (h.r.).

En el Tabla 4 se muestran las ecuaciones lineales y los coeficientes de correlación para los filtrantes almacenados a 70, 80, 90 y $100 \%$ de humedad relativa (H.R.), obtenidos mediante la grafica anterior. Los valores de $\mathrm{X}$ e Y indican el tiempo (días) y el contenido de antocianinas en el filtrante. Se observó que almacenamientos a 70 o $80 \%$ presentan comportamiento de degradaciones similares.

Tabla 4. Ecuaciones lineales y coeficiente de correlacion de los filtrantes almacenados bajo diferentes humedades relativas (h.r.).

\begin{tabular}{ccc}
\hline$\%$ HR & Ecuaciones Lineales & $\mathrm{r}^{2}$ \\
\hline 70 & $\mathrm{Y}=-0.0126 \mathrm{X}+7.9142$ & 0.9636 \\
80 & $\mathrm{Y}=-0.0149 \mathrm{X}+7.9237$ & 0.9639 \\
90 & $\mathrm{Y}=-0.0277 \mathrm{X}+7.9213$ & 0.9671 \\
100 & $\mathrm{Y}=-0.0291 \mathrm{X}+7.9200$ & 0.9717 \\
\hline
\end{tabular}

Según los resultados estadísticos, la degradación de las antocianinas fue significativa a los 12 días de almacenaje a condiciones de 70 y $80 \%$ de humedad relativa (H.R.); entre $6-12$ a $90 \%$ de humedad relativa (H.R.) y 6 días a $100 \%$ de humedad relativa (H.R); es decir el tiempo degradación fue menor a medida que aumenta la humedad relativa (H.R.) de almacenaje.

\section{B. Velocidad de degradación y Tiempo de Vida Media}

En el Tabla 5 se presenta las velocidades de la reacción (k) de degradación de las antocianinas. Al respecto, Schmalko et al. (2005) indican que al aumentar la actividad de agua aumenta el valor de la constante de velocidad específica.
En el Tabla 6 se muestra el tiempo (días) necesario para que la concentración de algunos reaccionantes disminuya a la mitad (Cantillo et al., 1994; citados por Moreno et al., 2002). Como se puede observar a condiciones de almacenaje de mayor humedad relativa (H.R.) se produce mayor degradación de antocianinas y menor tiempo de vida media.

Tabla 5. Velocidades de reacción de los filtrantes de maíz morado almacenados bajo diferentes humedades relativas (h.r.).

\begin{tabular}{cc}
\hline$\%$ HR & Velocidad de Reacción $(\mathrm{k})^{1}$ \\
\hline 70 & 0.0126 \\
80 & 0.0149 \\
90 & 0.0277 \\
100 & 0.0291 \\
\hline
\end{tabular}

${ }^{1}$ expresado en dias $^{-1}$

Tabla 6. Tiempo de vida media de antocianinas en filtrantes de maiz morado almacenados bajo diferentes humedades relativas (H.R.).

\begin{tabular}{cc}
\hline$\%$ H.R. & $\begin{array}{c}\text { Tiempo de vida media } \\
(\mathrm{t} 1 / 2) \text { en días }\end{array}$ \\
\hline 70 & 55.0 \\
80 & 46.5 \\
90 & 24.5 \\
100 & 23.8 \\
\hline
\end{tabular}

\section{Conclusiones}

El filtrante de maíz morado obtenido estuvo conformado por canela, clavo de olor, grano y coronta de maíz morado en proporciones de 1:1:10:88, respectivamente; debido a los resultados obtenidos en el análisis sensorial. La caracterización fisicoquímica realizada al filtrante de maíz morado reportó: humedad $7.9 \%$, carbohidratos $74.4 \%$, cenizas $2.4 \%$, grasa $0.2 \%$, proteína $5.1 \%$ y fibra $10.0 \%$ y actividad de agua 0.48 . La degradación de las antocianinas presentó una cinética de primer orden y la velocidad de degradación $(\mathrm{k})$ de las antocianinas fue de $0.0126,0.0149,0.0283$ y 0.0291 días $^{-1}$ y el tiempo de vida media fue de $55,46.5,24.5$ y 23.8 días a 70,80 , 90 y $100 \%$ de humedad relativa (H.R.), respectivamente. Los filtrantes presentaron mayor estabilidad en cuanto al color y degradación de antocianinas a condiciones de almacenaje a 70 u $80 \%$ de humedad relativa (H.R.).

\section{Literatura citada}

A.O.A.C. 1990. Official Methods of Analysis of the Association the Official Agricultural Chemists. De Board. USA.

A.O.A.C. 1995. Official Methods of Analysis of the Association the Official Agricultural Chemists. De Board. USA. 
A.O.A.C. 1997. Official Methods of Analysis of the Association the Official Agricultural Chemists. De Board. USA.

Araujo, J. 1995. Estudio del colorante de maíz morado (Zea mays L.) con el uso de enzimas. Tesis para optar el título de Magíster Scientiae. Universidad Nacional Agraria La Molina. Lima-Perú.

Calzada, J. 1982. Métodos estadísticos para la Investigación. Editorial Milagros. Lima-Perú.

Cancino, K. 2003. Influencia de la concentración del zumo en la deshidratación osmótica del yacón (Smallanthus sonchifolia Poepp. \& Endl.). Tesis para optar el título de Magíster Scientiae. Universidad Nacional Agraria La Molina. Lima-Perú.

Cheftel, J. y Cheftel, H. 1976. Introducción a la Bioquímica y Tecnología de los alimentos. Vol. I. Editorial Acribia S.A. Zaragoza-España.

Espinoza, P. 1995. Determinación de la Vida en Anaquel de Wafers (Obleas rellenas) mediante pruebas aceleradas. Tesis para optar el Título de Ingeniero en Industrias Alimentarias. Universidad Nacional Agraria La Molina. Lima-Perú.

Fennema, O. 2000. Química de los Alimentos. 2da Edición. Editorial Acribia S.A. Zaragoza-España.

Fernandez, N. 1995. Estudio de la Extracción y prepurificación de antocianinas de Maíz Morado (Zea mays L.). Tesis para optar el Título de Ingeniero en Industrias Alimentarias. Universidad Nacional Agraria La Molina. Lima-Perú.

Giusti, M. and Wrolstad, R. 2001. Characterization on measurement of anthocyanins by uv-visible spectroscopy. En: Current protocols in analytical Chemistry. Editorial John Wiley \& Sons. USA.

Labuza, T. 1979. A theoretical comparison on losses in food under fluctuating temperature sequences. Journal of Food Science, 44:1162-1168.

Lees, R. 1969. Manual de análisis de alimentos. Editorial Acribia-Zaragoza. España.

Martinez, E. 2004. Elaboración y determinación de Vida en Anaquel mediante pruebas aceleradas (ALST) del laminado de piña (Anonas sativus L.) coberturado con chocolate. Tesis para optar el título de Magíster Scientiae. Universidad Nacional Agraria La Molina. Lima-Perú.

Moreno, M.; Matos, A. y Belen, D. 2002. Degradación de betalaìnas en remolacha (beta vulgaris L.) estudio cinético. Revista Científica, 12(2): 133-136. MaracaiboVenezuela.

Robertson, G. 1993. Food Packaking. Marcel Dekker Inc. New York.

Schmalko, M.; Scipioni, P.; Ferreyra, D. y Alzamora, S. 2005. Efecto de la actividad del agua y la temperatura en la degradación de la clorofila y el color en Hojas de Yerba Mate. International Journal of Food Properties, 8: 313-322.
Vega, A.; Lara, E. y Lemus, R. 2006. Isotermas de absorción en Harina de Maìz (Zea mays L.). Ciencia y Tecnología Alimentaria, 26(4): 821-827. CampinasBrazil. 